\title{
AVALIAÇÃo de FILTROS dOMÉSTICOS COMERCIAIS PARA PURIFICAÇÃo de ÁGUAS E RETENÇÃo DE CONTAMINANTES INORGÂNICOS
}

\author{
Nilva Aparecida Ressinetti Pedro, Berenice Mandel Brígido, Maria Irene Cibela Badolato \\ Instituto Adolfo Lutz - Lab. I de Campinas - Rua São Carlos, 720 - 13035-420 - Campinas - SP \\ José Leopoldo Ferreira Antunes \\ Instituto Adolfo Lutz - Av. Dr. Arnaldo, 355 - 01246-902 - São Paulo - SP \\ Elisabeth de Oliveira \\ Instituto de Química - Universidade de São Paulo - Av. Lineu Prestes, 748 - 05508-900 - São Paulo - SP
}

Recebido em 24/4/96; aceito em 24/10/96

\begin{abstract}
EVALUATION OF DOMESTIC COMMERCIAL FILTERS TO WATERS PURIFICATION AND RETENTION OF INORGANIC CONTAMINATES. Twenty domestic commercial filters, in order to determine the percentual retention of color, turbidity, dry residue, bicarbonates, carbonates, total hardness, nitrogens, iron, chlorides, fluorides, and residual chlorine (parameters of food legislation) and sulphides in thirteen water samples proceeding from springs, wells, rivers, lakes, drinking patterns and standards, before and after purification were evaluated. The results showed that purifiers presented adequate retention for nitrates $(74.8 \pm 16.2 \%)$ and residual chlorine $(74.0$ $\pm 11.2)$ and medium retention for sulphides $(61.7 \pm 11.3)$; while porcelain plus activated carbon filters presented adequate retention for color $(90.0 \pm 19.7)$, turbidity $(76.4 \pm 18.4)$ and iron $(83.5$ \pm 15.1). Therefore the retention of carbonates, bicarbonates, total hardness, chlorides, dry residue, fluorides, ammonium nitrogens and nitrites was less than $10 \%$, and the values of pH didn't show significant variation, for all the filters studied.
\end{abstract}

Keywords: water; domestic filters; activated carbon.

\section{INTRODUÇÃO}

A água destinada ao consumo doméstico deve ser potável, isto é, isenta de contaminantes que possam comprometer a saúde do consumidor com qualquer moléstia de veiculação hídrica. As águas provenientes de poços e nascentes podem ser contaminadas tanto no ponto de captação, quanto no lençol de água, sendo que a principal fonte de contaminação são fossas negras. As águas tratadas podem ser contaminadas na captação, adução, tratamento, recalque ou instalações hidráulico- sanitárias prediais. Para melhorar a qualidade da água consumida, a população utiliza-se de filtros domésticos encontrados no comércio, os quais geralmente são cartuchos de porcelana porosa ou materiais similares e/ou carvão ativado, sendo que alguns possuem prata coloidal. A porcelana porosa ou similares é utilizada para retenção de matéria em suspensão que causa a turbidez da água. Muitas destas partículas, embora não sejam danosas por si próprias, podem afetar a qualidade da água indiretamente, agindo como veículos de transporte e liberação de outros contaminantes, como bactérias e vírus ${ }^{1,2}$. $\mathrm{O}$ carvão ativado é utilizado para retenção de gosto e odor, causados principalmente pelo cloro residual e compostos orgânicos (aldeídos, terpenos, compostos húmicos, etc.) em filtros domésticos e estações de tratamento, sendo que nenhum efeito nocivo foi descrito até o presente $\mathrm{s}^{3,4,5,6}$. O cloro é usado no tratamento da água de abastecimento público para vários propósitos, como desinfecção, controle de gosto e odor e remoção de $\operatorname{cor}^{7,8}$. A prata coloidal tem sido usada como bactericida ${ }^{9}$. A função conhecida dos filtros domésticos é a retenção de turbidez, de cloro residual e de bactérias, sendo importante acompanhar a medida de filtração (porcentagem de retenção) com outros contaminantes inorgânicos, que normalmente estão presentes nas águas, independente de sua origem.

O presente trabalho teve por finalidade a avaliação da eficácia dos principais tipos de filtros disponíveis no comércio, através da verificação da porcentagem de retenção de diferentes contaminantes como cor, turbidez, ferro, nitrogênios, etc., em diferentes amostras de águas naturais ou especialmente compostas para o experimento, que foram analisadas antes e após a filtragem.

Além dos parâmetros físico-químicos, a avaliação de potabilidade pela Norma Técnica de Alimentos ${ }^{10}$ (NTA 60 que dispõe sobre normas e padrões de potabilidade de águas de consumo alimentar, no Estado de São Paulo) e portaria no 36 do Ministério da Saúde ${ }^{11}$ (dispõe sobre normas e padrões de potabilidade de água destinada ao consumo humano, em todo o território nacional), inclui a análise microbiológica de retenção ou eliminação de bactérias de veiculação hídrica nocivas à saúde, o que não foi abordado no presente estudo, uma vez que existem várias pesquisas publicadas ${ }^{6,9,12,13}$ sobre este tópico, o que não ocorre com os parâmetros físico-químicos.

\section{MATERIAL E MÉTODOS}

\section{Material}

Foram utilizadas treze amostras de água, sendo dez amostras naturais coletadas na rede de abastecimento (tratadas), lagos, rios, poços e nascentes e três amostras padrões, sendo um padrão multielementar, um de nitrato e um de turbidez. O padrão multielementar contém concentrações conhecidas de dureza, nitrogênio amoniacal, nitrito, ferro, cloretos, fluoretos (preparado a partir da diluição de sais p. a. em água destilada) e de cloro residual pela diluição de hipoclorito de sódio $10 \%$ $(\mathrm{v} / \mathrm{v})$. O padrão de nitrato foi preparado de acordo com as Normas Analíticas do Instituto Adolfo $\mathrm{Lutz}^{14}$. O padrão de turbidez foi preparado de acordo com Pedro ${ }^{15}$, utilizando padrão de areia.

Alcalinidade de carbonatos e bicarbonatos, $\mathrm{pH}$ e resíduo seco foram apenas dosados na solução padrão multielementar. Para cor e sulfetos não foram preparadas amostras padrões pela dificuldade em obtê-las. 
Os elementos selecionados para avaliação são aqueles previstos pela Norma Técnica de Alimentos ${ }^{10}$ para a análise físicoquímica de águas, tendo sido incluída a determinação do teor de sulfetos, componente que produz odor. As concentrações desses elementos nas amostras naturais e nas amostras padrões, antes de serem filtradas, estão descritas nos quadros 1 e 2 .

Foram utilizados 20 filtros de 13 marcas diferentes comercialmente disponíveis na cidade de São Paulo - SP, cujas características principais estão descritas no quadro 3 . Os filtros de mesma marca foram de modelos diferentes. Levando-se em consideração o fato de que a capacidade de retenção dos filtros diminui após certo tempo de uso, todas as determinações foram efetuadas em um lote de filtros novos, especialmente adquiridos para esta finalidade.

\section{MÉTODOS}

As soluções padrões e as amostras naturais foram colocadas em um sistema de amostragem, conforme descrito por Pedro ${ }^{15}$ e filtradas sob pressão de $1,4 \mathrm{Kg} / \mathrm{cm}^{2}$, com vazão aproximada de 40 litros / hora, simulando o uso doméstico dos filtros.

As determinações de turbidez e cloro residual foram realizadas de acordo com Pedro ${ }^{15}$ e as demais determinações segundo as Normas Analíticas do Instituto Adolfo Lutz ${ }^{14}$, antes e após a filtração em cada um dos filtros estudados.

A análise das porcentagens de retenção para turbidez e cloro residual foram feitas de acordo com Pedro ${ }^{15}$ sendo considerada adequada a porcentagem de retenção de turbidez $\bullet 70 \%$ e de cloro residual de 90 ( \pm 6$) \%$. Para as demais determinações tomouse como referência ideal a mesma porcentagem de retenção da turbidez. Considerou-se como retenção média os valores entre 50 e $69 \%$ e retenção baixa para os valores abaixo de $50 \%$.

Para a análise estatística dos resultados, foi utilizado o programa de informática EPIDAT 1.0, da Organização Mundial da Saúde - OMS.

\section{RESULTADOS}

As porcentagens de retenção dos diversos elementos, para cada classe de filtro estudada, estão apresentadas na tabela 1.

Os gráficos 1 a 6 mostram as frequências de retenção de cor, nitrato, sulfetos, turbidez, ferro e cloro residual, respectivamente.

\section{DISCUSSÃO E CONCLUSÕES}

Pelos resultados obtidos podemos observar que não houve alteração significativa nos valores de $\mathrm{pH}$, representados nos quadros 1 e 2 em nenhuma das amostras estudadas. As porcentagens de retenção da dureza, resíduo seco, carbonatos, bicarbonatos, cloretos, fluoretos, nitrogênio amoniacal e nitrito para todos os filtros foram abaixo de $10 \%$, fato esperado uma vez que nos componentes dos filtros estudados não há nenhum material capaz de alterar o valor do $\mathrm{pH}$ ou reter os componentes acima citados.

Pela análise estatística da tabela 1, através do teste " $t$ " de Student, observou-se que os filtros de vela apresentaram retenção significativamente mais elevada que os demais tipos de filtros, para cor e turbidez $(\mathrm{p}<5 \%)$ e maior percentual de retenção para ferro, apesar de não ter configurado diferença significativa ( $\mathrm{p}>5 \%$ ), neste ítem, em relação aos cartuchos. Essa retenção é devida aos pequenos poros existentes na porcelana

Quadro 1. Características físico-químicas das amostras de água coletadas para avaliação dos procedimentos de filtragem.

\begin{tabular}{|c|c|c|c|c|c|c|c|c|c|c|}
\hline $\begin{array}{l}\text { Parâmetros físico- } \\
\text { químicos }\end{array}$ & Tratada & Lago & Rio & $\begin{array}{l}\text { Poço } \\
\text { am } 1\end{array}$ & $\begin{array}{c}\text { Amostras } \\
\text { Poço } \\
\text { am2 }\end{array}$ & $\begin{array}{l}\text { Nasc. } \\
\text { am } 1\end{array}$ & $\begin{array}{l}\text { Nasc. } \\
\text { am } 2\end{array}$ & $\begin{array}{l}\text { Nasc. } \\
\text { am } 3\end{array}$ & $\begin{array}{l}\text { Nasc. } \\
\text { am } 4\end{array}$ & $\begin{array}{l}\text { Nasc. } \\
\text { am } 5\end{array}$ \\
\hline Cor & 0 & 70 & 60 & 0 & 0 & 0 & 0 & 0 & 0 & 0 \\
\hline Turbidez (FTU) & 0,5 & 13,0 & 12,0 & 0,6 & 0,5 & 0,5 & 0,6 & 0,4 & 1,2 & 0,4 \\
\hline $\mathrm{pH}$ & 7,0 & 6,9 & 7,0 & 6,2 & 8,9 & 6,6 & 5,5 & 5,3 & 8,9 & 8,3 \\
\hline $\begin{array}{l}\text { Alcalinidade } \mathrm{CO}_{3}= \\
\left(\mathrm{mg} / \mathrm{l} \text { em } \mathrm{CaCO}_{3}\right)\end{array}$ & $<\mathrm{LD}$ & $<\mathrm{LD}$ & $<\mathrm{LD}$ & $<\mathrm{LD}$ & 1,0 & $<\mathrm{LD}$ & $<\mathrm{LD}$ & $<\mathrm{LD}$ & 100,0 & 28,0 \\
\hline $\begin{array}{l}\text { Alcalinidade } \mathrm{HCO}_{3}- \\
\left(\mathrm{mg} / \mathrm{l} \text { em } \mathrm{CaCO}_{3}\right)\end{array}$ & 11,0 & 29,0 & 36,0 & 17,0 & 64,0 & 22,0 & 3,0 & 15,0 & 270,0 & 149,0 \\
\hline $\begin{array}{l}\text { Dureza } \\
\left(\mathrm{mg} / \mathrm{l} \mathrm{em} \mathrm{CaCO}_{3}\right)\end{array}$ & 44,0 & 38,0 & 32,0 & 64,0 & 4,0 & 22,0 & 4,0 & 34,0 & 10,0 & 28,0 \\
\hline $\begin{array}{l}\text { Nitrogênio amoniacal } \\
(\mathrm{mg} / \mathrm{l} \text { em } \mathrm{N})\end{array}$ & 0,23 & $<\mathrm{LD}$ & 0,46 & $<\mathrm{LD}$ & $<\mathrm{LD}$ & $<\mathrm{LD}$ & $<\mathrm{LD}$ & 0,85 & 0,16 & $<\mathrm{LD}$ \\
\hline Nitrito (mg/l em N) & 0,03 & $<\mathrm{LD}$ & 0,04 & $<\mathrm{LD}$ & $<\mathrm{LD}$ & $<\mathrm{LD}$ & $<\mathrm{LD}$ & 0,03 & $<\mathrm{LD}$ & $<\mathrm{LD}$ \\
\hline Nitrato (mg/l em N) & 0,72 & $<\mathrm{LD}$ & 0,45 & 6,26 & 0,15 & 0,31 & 2,06 & 10,65 & $<\mathrm{LD}$ & $<\mathrm{LD}$ \\
\hline Ferro $(\mathrm{mg} / \mathrm{l})$ & 0,36 & 1,02 & 1,41 & 0,10 & 0,10 & 0,07 & 0,33 & 0,06 & 0,20 & 0,22 \\
\hline Cloretos (mg/l) & 8,2 & 3,1 & 5,8 & 9,5 & 7,1 & 3,7 & 5,1 & 11,5 & 816,0 & 598,0 \\
\hline Resíduo seco (mg/l) & 107,0 & 65,0 & 105,0 & 160,0 & 155,0 & 90,0 & 60,0 & 330,0 & 2120,0 & 1574,0 \\
\hline Fluoretos (mg/l) & 0,75 & $<\mathrm{LD}$ & $<\mathrm{LD}$ & $<\mathrm{LD}$ & 4,55 & $<\mathrm{LD}$ & $<\mathrm{LD}$ & $<\mathrm{LD}$ & $<\mathrm{LD}$ & $<\mathrm{LD}$ \\
\hline $\begin{array}{l}\text { Cloro residual } \\
\left(\mathrm{mg} / \mathrm{l} \text { em } \mathrm{Cl}_{2}\right)\end{array}$ & 0,47 & $<\mathrm{LD}$ & $<\mathrm{LD}$ & $<\mathrm{LD}$ & $<\mathrm{LD}$ & $<\mathrm{LD}$ & $<\mathrm{LD}$ & $<\mathrm{LD}$ & $<\mathrm{LD}$ & $<\mathrm{LD}$ \\
\hline Sulfetos $(\mathrm{mg} / \mathrm{l})$ & $<\mathrm{LD}$ & $<\mathrm{LD}$ & $<\mathrm{LD}$ & $<\mathrm{LD}$ & $<\mathrm{LD}$ & $<\mathrm{LD}$ & $<\mathrm{LD}$ & $<\mathrm{LD}$ & 14,5 & 3,2 \\
\hline
\end{tabular}

Am. $=$ amostra

Nasc. $=$ nascente

$\angle \mathrm{LD}=$ abaixo do limite de determinação do método; LD para alcalinidade de carbonatos e bicarbonatos e sulfetos:

$0,05 \mathrm{mg} / \mathrm{l}$; para os nitrogênios: $0,01 \mathrm{mg} / \mathrm{l}$ e para fluoretos e cloro residual livre: $0,02 \mathrm{mg} / \mathrm{l}$. 
Quadro 2. Características físico-químicas das amostras padrões de águas preparadas para avaliação dos procedimentos de filtragem.

\begin{tabular}{|c|c|c|c|}
\hline Parâmetros Físico - Químicos & $\begin{array}{c}\text { Padrão } \\
\text { multielementar }\end{array}$ & $\begin{array}{c}\text { Amostras } \\
\text { Padrão } \\
\text { de nitrato }\end{array}$ & $\begin{array}{c}\text { Padrão } \\
\text { de turbidez }\end{array}$ \\
\hline Turbidez ( FTU ) & - & - & 12,0 \\
\hline $\mathrm{pH}$ & 6,8 & - & - \\
\hline Alcalinidade $\mathrm{CO}_{3}^{\overline{\bar{N}}}\left(\mathrm{mg} / \mathrm{l}\right.$ em $\left.\mathrm{CaCO}_{3}\right)$ & 0 & - & - \\
\hline Alcalinidade $\mathrm{HCO}_{3}^{-}\left(\mathrm{mg} / \mathrm{l}\right.$ em $\left.\mathrm{CaCO}_{3}\right)$ & 79,0 & - & - \\
\hline Dureza $\left(\mathrm{mg} / \mathrm{l}\right.$ em $\left.\mathrm{CaCO}_{3}\right)$ & 300,0 & - & - \\
\hline Nitrogênio amoniacal (mg/l em N) & 0,78 & - & - \\
\hline Nitrito $(\mathrm{mg} / \mathrm{l}$ em N$)$ & 0,05 & - & - \\
\hline Nitrato $(\mathrm{mg} / \mathrm{l}$ em $\mathrm{N})$ & - & 8,27 & - \\
\hline Ferro $(\mathrm{mg} / \mathrm{l})$ & 0,60 & - & - \\
\hline Cloretos $(\mathrm{mg} / \mathrm{l})$ & 302,0 & - & - \\
\hline Resíduo seco (mg/l) & 1000,0 & - & - \\
\hline Fluoretos $(\mathrm{mg} / \mathrm{l})$ & 2,26 & - & - \\
\hline Cloro residual $\left(\mathrm{mg} / \mathrm{l}\right.$ em $\left.\mathrm{Cl}_{2}\right)$ & 0,92 & - & - \\
\hline
\end{tabular}

- não determinado

Quadro 3. Materiais filtrantes componentes dos filtros utilizados para avaliação da qualidade para fins de filtragens de águas.

\begin{tabular}{|c|c|c|}
\hline $\mathrm{N}^{\circ}$ do filtro & Materiais filtrantes & $\begin{array}{l}\text { Massa de carvão } \\
\text { ativado granular }(\mathrm{g})\end{array}$ \\
\hline Purificador 1 & 2 placas de resina + carvão ativado granular & 404 \\
\hline Purificador 2 & cascalho $*+$ carvão ativado granular & 230 \\
\hline Purificador 3 & cascalho $*+$ carvão ativado granular & 305 \\
\hline Purificador 4 & cascalho $*+$ carvão ativado granular & 200 \\
\hline Purificador 5 & porcelana com $\mathrm{Ag}+$ carvão ativado granular & 500 \\
\hline Purificador 6 & cascalho $*+$ carvão ativado granular & 340 \\
\hline Cartucho 7 & 2 placas de resina + carvão ativado granular & 122 \\
\hline Cartucho 8 & 2 placas de resina + carvão ativado granular & 270 \\
\hline Cartucho 9 & 2 placas de resina + carvão ativado granular & 300 \\
\hline Cartucho 10 & sílica com Ag + carvão ativado granular & 65 \\
\hline Cartucho 11 & sílica com Ag + carvão ativado granular & 120 \\
\hline Cartucho 12 & carvão ativado granular & 75 \\
\hline Cartucho 13 & 2 placas de resina + carvão ativado granular & 120 \\
\hline Cartucho 14 & 2 placas de resina + carvão ativado granular & 180 \\
\hline Vela 15 & vela de porcelana porosa dupla + carvão at. granular & 75 \\
\hline Vela 16 & vela de porcelana porosa & 0 \\
\hline Vela 17 & vela de porcelana porosa + carvão ativado granular & 50 \\
\hline Vela 18 & vela de porcelana porosa + carvão ativado granular & 7 \\
\hline Vela 19 & vela de material poroso + carvão ativado granular & 12 \\
\hline Vela 20 & vela de porcelana porosa + carvão ativado granular & 50 \\
\hline
\end{tabular}

* ou material filtrante similar

porosa, que se mostrou mais eficiente que o cascalho e outros materiais similares usados nos purificadores e as placas de resina porosa geralmente usadas nos cartuchos, na retenção das impurezas.

Os purificadores apresentaram retenção significativamente mais elevada que os demais tipos de filtros, para sulfetos e nitrato $(\mathrm{p}<5 \%)$ e apresentaram maior percentual de retenção para cloro residual, apesar de não ter configurado diferença significativa ( $\mathrm{p}>5 \%$ ), neste ítem, em relação aos cartuchos. Essa retenção é devida a grande massa de carvão ativado (330 $\pm 111 \mathrm{~g}$ ) utilizada nos purificadores, superior à massa média de carvão ativado utilizado nos cartuchos $(157 \pm 86 \mathrm{~g})$ e nas velas $(39 \pm 29 \mathrm{~g})$.

A classificação da retenção em adequada, média e baixa pode ser visualizada, para as três classes de filtros estudadas, nos gráficos 1 a 6 .

A análise dos gráficos 1,4 e 5 mostrou que, das seis velas estudadas, cinco (de porcelana porosa) apresentaram retenção adequada para cor, ferro e turbidez e apenas uma apresentou retenção média para cor e ferro e baixa para turbidez. Essa 
Tabela 1. Distribuição das porcentagens de retenção de componentes e contaminantes de águas, de acordo com o tipo de filtro.

\begin{tabular}{|c|c|c|c|c|c|c|c|c|c|c|c|c|}
\hline \multirow{3}{*}{ Tipo de filtro } & \multicolumn{12}{|c|}{ Componentes e contaminantes - Porcentagem de retenção } \\
\hline & \multicolumn{2}{|c|}{ Cor } & \multicolumn{2}{|c|}{ Cloro residual } & \multicolumn{2}{|c|}{ Sulfetos } & \multicolumn{2}{|c|}{ Turbidez } & \multicolumn{2}{|c|}{ Ferro } & \multicolumn{2}{|c|}{ Nitrato } \\
\hline & M & DP & M & DP & M & DP & M & DP & M & DP & M & DP \\
\hline $\begin{array}{l}\text { Purificadores } \\
\text { (F1 a F6) }\end{array}$ & 41,8 & 26,2 & 84,0 & 11,2 & 61,7 & 11,3 & 41,7 & 24,9 & 58,8 & 15,2 & 74,8 & 16,2 \\
\hline $\begin{array}{l}\text { Cartuchos } \\
\text { (F7 a F14) }\end{array}$ & 34,1 & 21,4 & 65,7 & 21,6 & 34,9 & 20,1 & 37,9 & 22,5 & 64,8 & 21,4 & 45,6 & 25,7 \\
\hline $\begin{array}{l}\text { Velas } \\
\text { (F15 a F20) }\end{array}$ & 90,0 & 19,7 & $50,9^{*}$ & $31,1^{*}$ & 26,3 & 16,9 & 76,4 & 18,4 & 83,5 & 15,1 & 24,8 & 28,6 \\
\hline
\end{tabular}

* Não foi considerado F16 por não conter carvão ativado; $\mathrm{M}$ = média e DP = estimativa do desvio padrão

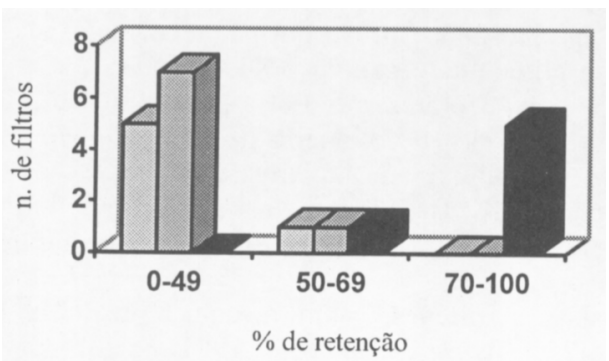

Gráfico 1. Porcentuais de retenção de cor.

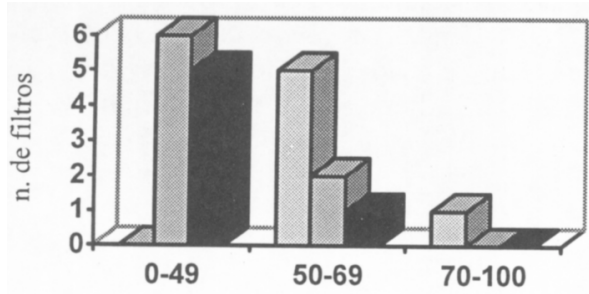

Gráfico 3. Porcentuais de retenção de sulfetos.

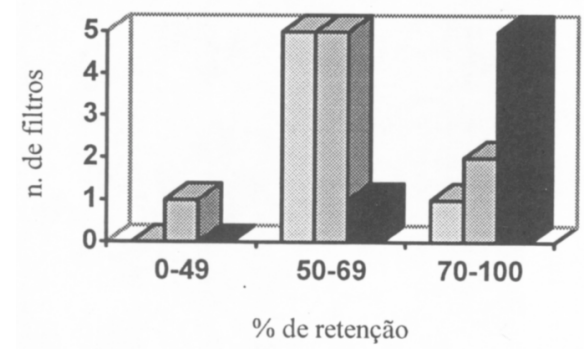

Gráfico 5. Porcentuais de retenção de ferro.

Legenda: Purificador

Cartuchos

última vela não era de porcelana porosa e sim de outro material, não identificado, semelhante a resina e possivelmente com poros maiores e menor eficácia do que a porcelana. Nenhuma vela apresentou retenção adequada para nitrato e sulfetos (gráficos 2 e 3) e apenas uma para cloro residual (gráfico 6), devido à pequena massa de carvão ativado existente nas mesmas.

Pelos gráficos 2 e 6 observou-se que, dos seis purificadores estudados, cinco apresentaram retenção adequada e um retenção média para nitrato, três retenção adequada e três retenção média para cloro residual, possivelmente devido à baixa quantidade de sítios de adsorção do carvão ativado utilizado em alguns deles. Nenhum purificador apresentou retenção adequada para cor (gráfico 1) e apenas um para sulfetos, turbidez e

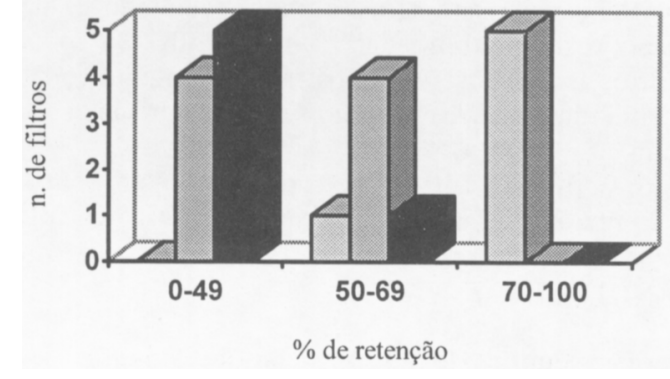

Gráfico 2. Porcentuais de retenção de nitrato.

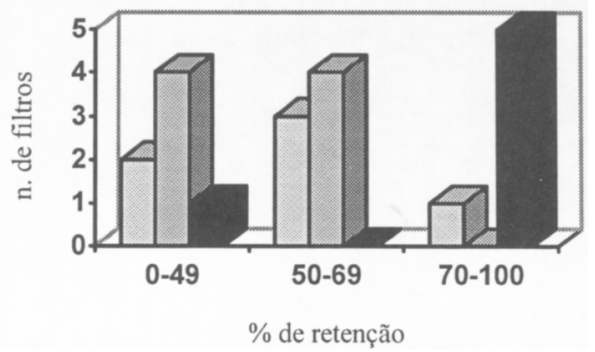

Gráfico 4. Porcentuais de retenção de turbidez.

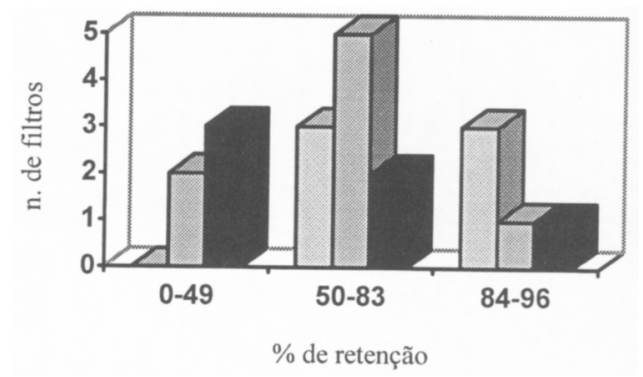

Gráfico 6. Porcentuais de retenção de cloro residual.

ferro (gráficos 3, 4 e 5), mostrando que o material filtrante utilizado é menos eficiente que a porcelana porosa.

Apenas um cartucho apresentou retenção adequada para sulfetos e cloro residual (gráficos 3 e 6) e dois para ferro (gráfico 5). Para os outros componentes estudados nenhum cartucho foi adequado. Isto pode ser explicado pela análise dos componentes dos cartuchos, que são de placas de resina ou sílica granulada, materiais que mostraram pior desempenho quando comparados à porcelana porosa. Além disso, os cartuchos continham menor massa de carvão ativado que os purificadores.

Taylor e col $^{16}$. testaram quatro cartuchos contendo carvão ativado com massa média de $406 \pm 150 \mathrm{~g}$, semelhante à 
existente nos purificadores testados; para avaliar a retenção de cloro residual em meses de baixas temperaturas $\left(2\right.$ a $\left.20^{\circ} \mathrm{C}\right)$, encontrando porcentagem de retenção de 57,3 $\pm 6,0 \%$ e em meses de temperaturas mais altas $\left(9\right.$ a $\left.27^{\circ} \mathrm{C}\right)$, encontrando porcentagem de retenção de $86,4 \pm 9,7 \%$, resultado este semelhante para os purificadores do presente trabalho.

Com relação aos demais elementos testados, não foram encontrados na literatura trabalhos específicos sobre avaliação da qualidade dos diversos tipos de filtros domésticos.

Com base nas porcentagens de retenção encontradas podemos concluir que os purificadores são adequados para retenção de cloro residual e nitrato e as velas de porcelana porosa são adequadas para retenção de cor, turbidez e ferro, quanto ao aspecto físico-químico.

A classificação das amostras filtradas segundo os padrões físico-químicos de potabilidade ${ }^{10,11}$, foi de $50 \%$ de aprovação, para os filtros estudados. As amostras provenientes do lago e rio não foram classificadas por não serem destinadas ao consumo humano.

Portanto, o tipo de filtro a ser adquirido pelo consumidor deve ser compatível às características da água a ser filtrada.

\section{REFERÊNCIAS}

1. American Public Health Association, Standart Methods for the Examination of Water and Wastewater, 14- ed., Washington, 1975, 1193 p.

2. Willians, R. B. e Culp, G. L.; Handbook of Public Water Systems, Van Nostrand Reinhold ed., New York, 1를. ed., 1986, 1113 p.

3. Hyndshaw, A. Y.; J. Am. Water Works Association 1972 , 64, 309.
4. Morris, G. e Newcombe, G.; J. of Colloid and Interface Science 1993, 159, 413.

5. Stevens, A. A.; Slocum, C. J.; Seezer, D. R. e Robeck, G. G.; J. Am. Water Works Association 1976, 68, 615.

6. Brewer, W. S. e Carmichael, W. W.; J. Am. Water Works Association 1979, 71, 738 .

7. Kreft, P.; Umphres, M.; Hand, J. M.; Tote, C.; Mcguire, M. J. e Trussel, R. R.; J. Am. Water Works Association 1985, 77, 38 .

8. Wartchow, D.; Freitas, A. F. R. e Luca, S. J.; Rev. Dep. Águas e Esgoto 1984, 136, 57.

9. Bell Jr., F. A.; J. Am. Water Works Association 1991, 83, 74.

10. São Paulo, Leis, decretos, etc.-Decreto no 12.486 de 20 de outubro de 1978. Diário Oficial, São Paulo, 21 de out. 1978, p. 280-283. Aprova normas técnicas especiais relativas a alimentos e bebidas (NTA 60).

11. Brasil, Leis, decretos, etc. Portaria no 36/GM do Ministério da Saúde de 19 de janeiro de 1990, Diário Oficial da União, Brasília, 23 de janeiro de 1990.

12. Camper, A. K.; Broadaway, S. C.; LeChevallier, M. W. e McFeters, G. A.; J. Am. Water Works Association 1987, 79, 70.

13. Servais, P.; Billen, G.; Ventresque, C. e Babla, G. P., J. Am. Water Works Association 1991, 83, 62.

14. Instituto Adolfo Lutz, Normas Analíticas do Instituto Adolfo Lutz: Métodos Químicos e Físicos para Análise de Alimentos, São Paulo, 3aㅡ ed. 1985, 302, 330.

15. Pedro, N. A. R.; Dissertação de Mestrado, Instituto de Química, Universidade de São Paulo, 1990, 81 p.

16. Taylor, R. H.; Allen, M. J. e Geldreich, E. E.; J. Am. Water Works Association 1979, 71, 577. 